\title{
The Effects on the Response of Metal Oxide and Fluorite Single Crystal Electrodes and the Equilibration Process in the Interfacial Region
}

\author{
Tajana Preočanin, * Danijel Namjesnik, Tin Klačić, Petar Šutalo
}

\author{
Department of Chemistry, Faculty of Science, University of Zagreb, Horvatovac 102a, HR-10000 Zagreb, Croatia \\ * Corresponding author's e-mail address: tajana@chem.pmf.hr \\ RECEIVED: August 31, 2017 * REVISED: September 10, 2017 * ACCEPTED: September 11, 2017
}

THIS PAPER IS DEDICATED TO PROF. MIRJANA METIKOŠ-HUKOVIĆ ON THE OCCASION OF HER BIRTHDAY

\begin{abstract}
Inner surface potential, one of the most important variables affecting the interfacial equilibrium of metal oxide aqueous systems, obtained by means of single crystal electrode gives valuable information about electrical charging of the metal oxide/aqueous electrolyte solution interfaces. The influence of the potential determining ions and ionic strength on the measured electrode potential as well as time of the equilibration, direction of the titration and the effect of the magnetic stirring enables the critical examination of the processes which take place during the interfacial equilibrium. For that purpose, the selected metal oxides (hematite, ceria, sapphire, and rutile) and fluorite single crystal electrodes were examined.
\end{abstract}

Keywords: single crystal electrode, inner surface potential, electrical double layer, metal oxide, fluorite, surface complexation, stirring effect.

\section{INTRODUCTION}

$\mathbf{T}$ HE understanding of surface complexation at metal oxide/aqueous electrolyte solution is important to determine the chemical and electrostatic forces involved in ion retention as well as to provide a framework that allows such processes to be modeled. Formation of electrical interfacial layer (EIL) at the contact of metal oxide particles and aqueous electrolyte solution has been subject of numerous theoretical as well as experimental studies. ${ }^{[1,2]}$ The reactions of ions with metal oxide surface cause the formation of the charged surface groups and consequently the distribution of ions within metal oxide/solution interface. Additionally, due to rearrangement of water dipoles near the solid surface the interfacial water has more structured hydrogen bonding network than bulk water. ${ }^{[3]}$ Structure of EIL is commonly described by several layers and planes characterized by surface charge densities and surface potentials. ${ }^{[4-6]}$ Within the solid phase the potential is assumed to be constant and equal to the inner surface potential $\psi_{0}$ corresponding to the 0 -plane that divides the solid from the liquid phase and where the charged surface groups are located. Within the interfacial layer the value of the potential decreases from the solid surface to the bulk of the solution. The bulk of the solution is commonly taken as a reference point that has a zero value of the electrostatic potential (Figure 1).

For metal oxide surfaces $\mathrm{H}^{+}$and $\mathrm{OH}^{-}$are the dominant potentially determining ions, however, binding and

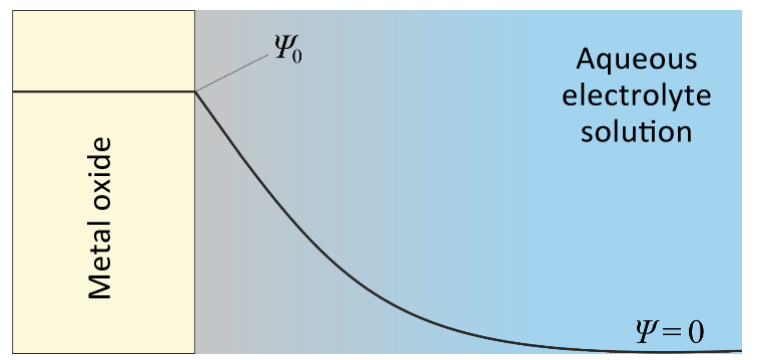

Figure 1. Within the interfacial layer the value of the potential decreases from the solid surface to the bulk of the solution. 


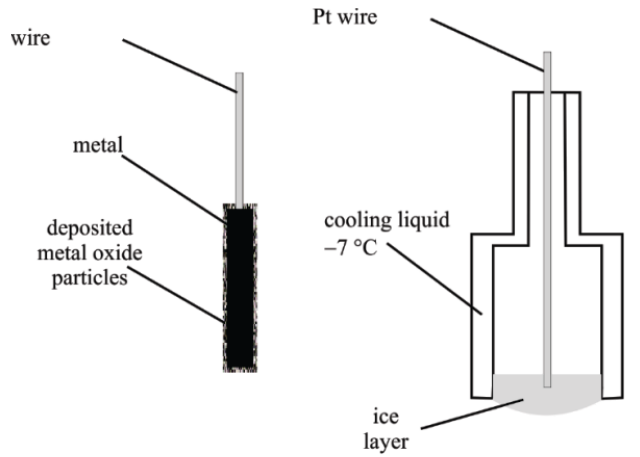

(a)

(b)

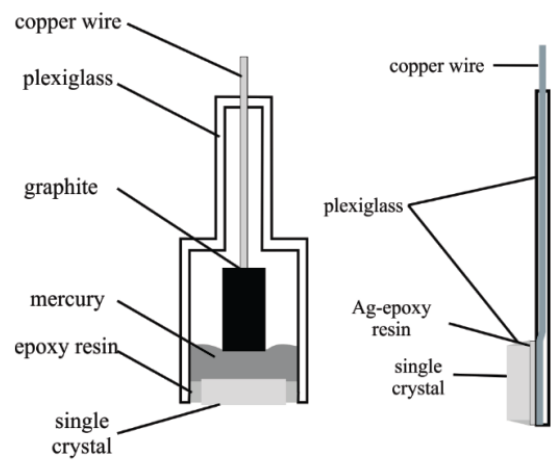

(c)

(d)

Figure 2. Various designs of electrodes for determining the surface potential at solid-water interfaces: (a) coated wire electrode, (b) ice electrode, (c) single crystal electrode, (d) single crystal electrode with conductive paint.

distribution of other counterions should be taken into account. According to the simplest model of surface complexation all surfaces have a single site type, each site can undergo protonation or deprotonation reaction, charges are always expressed as integers and strict distinction between inner- and outer-sphere complexes exists. The inner surface potential $\psi_{0}$ directly affects the state of ionic species ( $\equiv \mathrm{MO}^{2}$ and $\equiv \mathrm{MOH}^{2+1}$, where $\mathrm{M}$ represents metal atoms) bound to a certain interfacial plane and is thus an essential parameter governing interfacial equilibrium described by thermodynamic equilibrium constant ${ }^{[7,8]} K^{\circ}$

$$
\begin{aligned}
& \equiv \mathrm{MO}^{2}+\mathrm{H}^{+} \rightleftarrows \equiv \mathrm{MOH}^{2+1} ; \\
& K^{\circ}=\exp \left(F \Psi_{0} / R T\right) \cdot \frac{\left\{\equiv \mathrm{MOH}^{z+1}\right\}}{\left\{\equiv \mathrm{MO}^{2}\right\} \cdot a_{\mathrm{H}^{+}}}
\end{aligned}
$$

where $a_{\mathrm{H}+}$ denotes the activity of oxonium ions in the bulk of the solution. Curly brackets denote surface concentrations (amount of surface species per surface area). The exponential term in Equation (1) was derived from the activity coefficients of charged surface species. The overall inner surface potential depends not only on the number and charge of the surface sites, but also on the ratio between positively and negatively charged surface sites $\left\{\equiv \mathrm{MOH}^{2+1}\right\} /\left\{\equiv \mathrm{MO}^{z}\right\}$

The metal oxide/aqueous electrolyte interface is in the state of point of zero potential $\left(\mathrm{pH}_{\mathrm{pzp}}\right)$ when the value of the inner surface potential $\psi_{0}$ is equal to zero. In this case the activity coefficients of charged surface species are one, and electrostatic interactions diminish. The direct measurements of the value of the point of zero potential is impossible. The easiest method is to identify the point of zero potential with the point of zero charge $\left(\mathrm{pH}_{\mathrm{pzc}}\right)$ or the isoelectric point $\left(\mathrm{pH}_{\mathrm{iep}}\right)$. However, the more accurate methods for determination of the point of zero potential were proposed. Cyclic titration from acidic to basic $\mathrm{pH}$ (alkalimet- ric), and the reverse (acidimetric), often exhibits hysteresis, ${ }^{[9]}$ which was exploited for determination of the point of zero potential.[10] In the absence of hysteresis and counterion association another method was proposed which is based on a common-intersection point of measured electrode potential functions at different ionic strengths. ${ }^{[11]}$

Last decades numerous attempts (Figure 2) were made to measure the inner surface potential of metal oxides, an important quantity that affects the state of charged surface sites through their interfacial activity coefficients and distribution of ions within electrical interfacial layer. First attempts were to produce the electrode made by deposition or compression of fine metal oxide colloid particles on the metal wire (Figure 2a). ${ }^{[12-17]}$ Such electrodes are porous and have high electrical resistivity what make surface potential measurement uneasy and unreliable. The next attempt was to use semiconducting materials such as an ion sensitive field-effect transistor (ISFET). ${ }^{[18-25]}$ The ISFET electrode was already used as a sensitive electronic device for determining the concentration of $\mathrm{H}^{+}$ions in the solutions. Twelve years ago, the first metal oxide single crystal electrode ( $\mathrm{SCrE}$ ) was constructed (Figure $2 \mathrm{c})^{[26]}$ and since then have been developed (Figure 2d) ${ }^{[9,27-42]} \mathrm{A}$ special case of the single crystal electrode is the ice electrode, ${ }^{[43,44]}$ i.e. a metal wire covered by a thin layer of ice (Figure $2 b$ ).

The detail description and development of devices for surface potential measurements is given below.

\section{Ion Sensitive Field-Effect Transistor (ISFET)}

Shortly after the introduction of the first ISFET devices their sensitivity to $\mathrm{pH}$ was noticed and as such they are used as a conventional glass electrode (i.e. for measuring the $\mathrm{pH}$ of a solution). ${ }^{[18-25]}$ An electrode based on the ISFET concepts consists of a metal attached to a semiconductor (e.g. Si) 
Table 1. The sensitivity of the ISFET electrodes.

\begin{tabular}{ccc}
\hline ISFET electrode & $\left(\frac{\mathrm{d} \Psi_{0}}{\mathrm{dpH}}\right)_{25^{\circ} \mathrm{C} / \mathrm{mV}}$ & Reference \\
\hline Nernstian potential & -59.2 & theoretical \\
$\mathrm{TiO}_{2}$ & -56.2 & {$[21]$} \\
$\mathrm{Al}_{2} \mathrm{O}_{3}$ & -48.5 & {$[19]$} \\
$\mathrm{SiO}_{2}$ & -45.0 & {$[20]$} \\
\hline
\end{tabular}

whose surface is covered by the phase of interest, an insulator (e.g. $\mathrm{SiO}_{2}$ ) which is in contact with an electrolyte, i.e.

metal | semiconductor | insulator | aqueous

electrolyte solution | reference electrode

when the ion concentration (such as $\mathrm{H}^{+}$) changes, the current through the ISFET will change accordingly. From the response times and sensitivity of ISFET electrodes it was concluded that surface reactions determine their response. ${ }^{[23]}$ Surface reactions lead to surface charging and a distribution of ions in the electrolyte solution near the oxide surface. The mechanism responsible for the metal oxide surface charge was described by the site binding model, i.e. the equilibrium between the metal oxide surface sites and the $\mathrm{H}^{+}$ions in the solution. ${ }^{[4]}$ The measured electrode potential of the ISFET electrodes is particularly sensitive to the potential drop at the semiconductor/electrolyte interface which is developed due to metal oxide surface protonation and deprotonation. The measured flat-band voltage of the semiconductor is sensitive to the potential difference at the metal oxide/electrolyte interface. The ISFET electrode is a sensitive electronic device for determining the concentration of $\mathrm{H}^{+}$ions and also for the study of equilibrium processes at the metal oxide/aqueous electrolyte interfaces. ISFETs have been successfully used for $\mathrm{SiO}_{2}$, [21] $\mathrm{TiO}_{2}$, [22] $\mathrm{Al}_{2} \mathrm{O}_{3}{ }^{[20]}$ surfaces. The sensitivity of the ISFET electrode (the dependency of surface potential) to $\mathrm{pH}$ (compared to the Nernstian slope) is given in Table 1. ISFET sensors exhibit some advantages compared to glass electrodes such as their small size, robustness, fast response, low impedance, easy storage and cleaning. ${ }^{[25]}$

\section{Polycrystalline Metal Oxide Coated Wire Electrode}

The surface potential of metal oxide/electrolyte interfaces has also been examined by using a coated wire electrode (Figure 2a), that is, a metallic wire covered by metal oxide colloid particles, i.e.

metal | layer of colloid particles | aqueous electrolyte solution | reference electrode
Measurements of open circuit potential (OCP) using $\mathrm{Ag} / \mathrm{AgCl},{ }^{[17]} \mathrm{Pt} /$ hematite $\left(\alpha-\mathrm{Fe}_{2} \mathrm{O}_{3}\right),{ }^{[16]} \mathrm{Ti}^{1} / \mathrm{TiO}_{2},{ }^{[15]} \mathrm{ZrO}_{2},{ }^{[14]}$ $\mathrm{Pd} / \mathrm{PdO}{ }^{[12]}$ or $\mathrm{IrO}_{2}{ }^{[12]}$ electrodes are good illustrations of this method. The main problem with these electrodes is the porosity of the oxide layer, which does not avoid direct contact of the aqueous electrolyte solution with the metal. With sufficient porosity, the measured potential is determined by the redox equilibrium at the metal/aqueous electrolyte solution interface and by the concentrations of interacting ions that depend on the solubility of the metal oxide. In fact, these electrodes behave as electrodes of the second kind. ${ }^{[45]}$ The measured slope of the $\psi_{0}(\mathrm{pH})$ or $\psi_{0}(\mathrm{pAg})$ functions was found to be Nernstian. ${ }^{[12-19]}$ Such a result would also be in agreement with a surface complexation model involving a sufficiently high number of reactive surface sites.

\section{Single Crystal Electrode}

The problems arising from metal oxide colloid particle layer porosity are eliminated by the construction of single crystal electrode. The idea of using a single crystal as an electrode goes back several decades to when Quinn et a ${ }^{[46]}$ measured the current and voltage from an electrode made from a hematite single crystal. The first single crystal electrode, constructed for the purpose of surface potential evaluation, was the "ice electrode". ${ }^{[43,44]}$ A compact ice layer was formed on the platinum (Figure $2 \mathrm{~b}$ ) and the measured potential was the result of reversible interfacial reactions at the water/ice interface, i.e.

$\mathrm{Pt} \mid$ ice layer $\left(-2{ }^{\circ} \mathrm{C}\right) \mid$ aqueous electrolyte solution $\left(0^{\circ} \mathrm{C}\right) \mid$ reference electrode

The temperature inside the electrode was $-2{ }^{\circ} \mathrm{C}$, while the electrolyte solution was kept at $0{ }^{\circ} \mathrm{C}$, so that an ice layer was formed on the platinum plate. In the acidic region, fast equilibration of the electrode was observed. The slope of the $\psi_{0}(\mathrm{pH})$ function was found to be between -40 and $-46 \mathrm{mV}$, i.e. lower in magnitude than the Nernstian potential $\left(-54.2 \mathrm{mV}\right.$ at $\left.0{ }^{\circ} \mathrm{C}\right)$. Moreover, the $\psi_{0}(\mathrm{pH})$ function was not linear and the point of zero potential was reported to be at $\mathrm{pH}_{\mathrm{pzp}}=4.4$. The results were explained on the basis of the surface complexation model (SCM), ${ }^{[7]}$ assuming an amphoteric nature of ice surface $\mathrm{OH}$ groups.

Following the idea of ice electrode, the first single crystal electrode was built from hematite single crystal larger than few millimeters embedded in Plexiglas holder. Several crystal planes were exposed to the aqueous electrolyte solution. The electrical contact with the connecting metal wire made possible the measurement. ${ }^{[26]}$ The construction of single crystal electrodes has been improved by using the single crystal and exposing only one crystal plane to the aqueous electrolyte solution (Figure $2 \mathrm{c}$ ). 
Graphite(s) | $\mathrm{Hg}(\mathrm{I}) \mid$ single crystal | aqueous electrolyte solution | reference electrode

Recently a new version of the single crystal electrode was constructed without potentially dangerous mercury and provides a good electrical contact of the metal wire with the metal oxide surface through a conductive paint (Figure 2d), i.e.

$\mathrm{Cu}(\mathrm{s})$ | conductive paint | single crystal | aqueous electrolyte solution | reference electrode

The potential of the metal oxide single crystal electrodes depends on $\mathrm{pH}$ due to equilibrium surface reactions, i.e. adsorption and release of $\mathrm{H}^{+}$ions on/from the surface. It was found that the potential of the single crystal silver halide electrodes, $\mathrm{AgCl}\left[{ }^{[47-49]}\right.$ and $\mathrm{AgBr},{ }^{[50,51]}$ depends on the activities of $\mathrm{Ag}^{+}$and $\mathrm{Cl}^{-}$or $\mathrm{Br}^{-}$ions, respectively.

Under equilibrium conditions (without applied external voltage and when electrical current decreases towards zero) the inner electrical potential within the solid phase is assumed to be constant and equal to the inner surface potential, $\Psi_{0}$ (Figure 1). This Galvani potential is defined as the difference in the electrostatic potential felt by a given ion in the interior of both phases. It is equivalent to the electric work required to transfer this ion from the interior of one phase to the interior of the other. ${ }^{[52]}$ The values of metal oxide inner surface potential are evaluated from the measured open circuit potential of a SCrE. The open circuit potential is measured with respect to the reference electrode using a $\mathrm{pH}$-meter (i.e. high resistance voltmeter). In the electrical circuit only the potential at single crystal/aqueous electrolyte solution interface is sensitive to the concentration of potential determining ions. The value of the inner surface potential could be obtained from the measured open circuit potential $(E)$ by

$$
\psi_{0}=E-E_{\mathrm{T}}
$$

where the constant term denoted as $E_{\mathrm{T}}$ represents sum of all potential jumps that are independent of the activities of potential determining ions. There are several proposed methods for the evaluation of the surface potential from measured electrode potential. ${ }^{[53]}$ It is enough to determine the point of zero potential $\mathrm{pH}_{\mathrm{pzp}}$ at which the value of inner surface potential is zero. In the absence of specific ion adsorption and symmetrical association of counterions the point of zero potential corresponds to point of zero charge or isoelectric point. The point of zero charge could be obtained by potentiometric acid-base titrations ${ }^{[2,54]}$ or potentiometric mass titration originally developed by Noh and Schwarz ${ }^{[55]}$ and refined by Kallay and Žalac, ${ }^{[56]}$ while the isoelectric point can be obtained by electrokinetic techniques such as electrophoresis or streaming potential measurements. ${ }^{[57,58]}$ In the case of specific adsorption or unsymmetrical association of counterions, the point of zero potential could be obtained by several proposed experimental methods. As one example the appearance of hysteresis of electrode potentials during acid base titration, ${ }^{[10]}$ the method of a common intersection point of measured electrode potential functions at different ionic strengths, ${ }^{[11]}$ a saddlelike surface potential function $\psi_{0}(\mathrm{pH}) .{ }^{[53]}$

During the potential measurements with the metal oxide SCrE the electrolyte solution is occasionally stirred, liquid flowing along a solid crystal plane could affects the distribution of ions and arrangements of water molecules within the EIL. The influence of stirring of the electrolyte solution on the measured electrode potentials can be expressed as a difference in measured SCrE potential with stirring-off and stirring-on conditions

$$
\Delta E_{\text {stirr }}=E_{\text {stirroff }}-E_{\text {stirron }}
$$

where $\Delta E_{\text {stirr }}$ is "stirring potential". The effect of stirring on the (glass) electrode potential was known from previous research. ${ }^{[59-61]}$ However, theoretical explanation of this effect never converged into the wide accepted model. The general lack of interest for these phenomena is mostly due to the fact that the magnitude of this effect is negligible. However, it was found that the effect of stirring depends on the composition of the measuring solution i.e. concentration of potential determining ions as well as ionic strength and temperature. For the purposes of more precise SCrE potential measurements, necessary for the investigation of the mechanisms contributing to the electrode potentials and the description of the charging mechanisms at the electrical interfacial layer, the effect of stirring was examined.

In this work, we present the electrical properties of the metal oxide and fluorite single crystal electrodes, the limits and the possibilities of the measurements of the inner surface potential. For that purpose, the improved version of single crystal electrode as well as high impedance voltmeter were constructed. The properties of SCrE and mechanism of electrical charging were investigated by measuring the impedance, electrical current and the electrode potential of SCrE in respect to silver/silver chloride reference electrode under equilibrium and non-equilibrium conditions. The experiments were designed to monitor electrode potential, time of equilibration, the effect of stirring and possible hysteresis as a function of $\mathrm{pH}$, ionic strength and stirring speed. Data analysis gives the better understanding of the processes which take place during potentiometric acid base titration by means of metal oxide and fluorite single crystal electrodes.

\section{EXPERIMENTAL}

\section{Crystals and Solutions}

The hematite (0001), rutile (100), rutile (110), rutile (001), quartz (0001), ceria (111), ceria (100), sapphire (0001), and fluorite (111) single crystals were obtained from MaTeck, Jülich 
(Germany), and SurfaceNet GmbH (Germany). The dimensions of single crystals were: $5 \mathrm{~mm}$ by $5 \mathrm{~mm}$ (hematite and ceria), and $10 \mathrm{~mm}$ by $10 \mathrm{~mm}$ (quartz and rutile). All crystals were $0.5 \mathrm{~mm}$ thick, EPI-polished on one side. The samples were cleaned as described earlier. ${ }^{[41]}$ All solutions were prepared using deionized and decarbonized water (> $18 \mathrm{M} \Omega \mathrm{cm}$ ) and analytical grade chemicals. The aqueous electrolyte solutions were prepared by dilution of standard acid and base solutions $(\mathrm{NaOH}, \mathrm{HCl}$ : Fluka, fixanal, $\left.c=0.1 \mathrm{~mol} \mathrm{dm}^{-3}\right)$; and dissolution of weighted ammount of salt ( $\mathrm{NaCl}$ : Fluka, puriss p.a.) to prepare solutions of aimed ionic strengths $\left(0.001 \mathrm{~mol} \mathrm{dm}^{-3}\right.$, and $\left.0.01 \mathrm{~mol} \mathrm{dm}^{-3}\right)$.

\section{The Construction of SCr Electrodes}

The metal oxide single crystal (hematite, ceria, sapphire, and rutile) and fluorite single crystal electrode were constructed by using a conductive paint (Figure $2 \mathrm{~d}$ ).

\section{Potentiometric Titrations}

The effect of potential determining ions $(\mathrm{pH})$, ionic strength and stirring speed on all constructed metal oxide and fluorite single crystal electrodes were examined.

A typical acid-base potentiometric titration with $\mathrm{SCrE}$ is shown at Figure 3. The $\mathrm{pH}$ was controlled by adding $0.1 \mathrm{~mol} \mathrm{dm}^{-3} \mathrm{NaOH}$ to the $0.01 \mathrm{~mol} \mathrm{dm}^{-3} \mathrm{HCl}$ solution. The volumes of $\mathrm{NaOH}$ added to the solution were not calculated in advance, neither were of critical importance to be noted. Since the solution's chemical equilibrium is negligibly impacted by the reactions on the crystal surface, the solution's composition can be controlled solely by monitoring $\mathrm{pH}$ and controlling successive volumes of $\mathrm{NaOH}$ added, aiming the desired $\mathrm{pH}$ values on the point-by-point base. During measurements, the aqueous electrolyte solution was mixed with a magnetic stirrer (728 Magnetic Stirrer, Metrohm, Switzerland), thermostated (F12-ED, Julabo, Germany) at $(25.0 \pm 0.1){ }^{\circ} \mathrm{C}$ and kept under argon

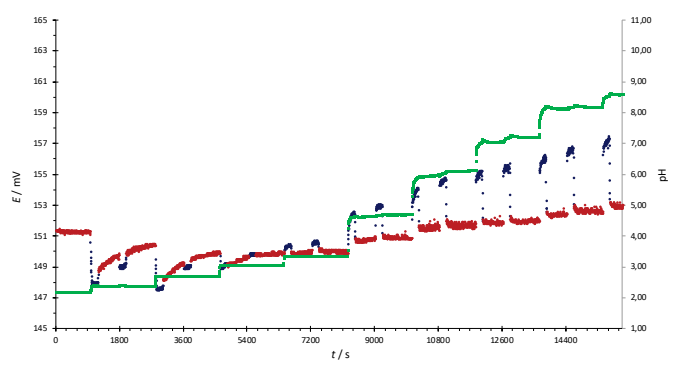

Figure 3. Potentiometric titration of hematite, $\alpha-\mathrm{Fe}_{2} \mathrm{O}_{3}$ (0001) SCrE: electrode potential of SCrE with stirring off $(\bullet)$, with stirring on $(\bullet)$, and $\mathrm{pH}$ obtained by glass electrode $(\bullet)$. The $\mathrm{pH}$ was controlled by adding $0.1 \mathrm{~mol} \mathrm{dm}^{-3} \mathrm{NaOH}$ to the $0.01 \mathrm{~mol} \mathrm{dm}^{-3} \mathrm{HCl}$ solution. During measurements, the aqueous electrolyte solution was mixed with a magnetic stirrer, thermostated at $(25.0 \pm 0.1)^{\circ} \mathrm{C}$ and kept under argon atmosphere. atmosphere. The entire measuring system was placed in a Faraday cage so that external electrical interferences were avoided. Electrode potential of single crystal electrodes $(E / \mathrm{mV})$ relative to reference electrode $\mathrm{Ag} / \mathrm{AgCl} / \mathrm{KCl}\left(3 \mathrm{~mol} \mathrm{dm}^{-3}\right)$ was measured using a pH-meter (Metrohm 827 or constructed high impedance voltmeter). The $\mathrm{pH}$ was measured by separated glass electrode (Metrohm Microelectrode). During titrations, the measured signals were collected every $4 \mathrm{~s}$ by using RS232 DataLogger 2.7 (Eltima Software), coupled with customized MS Excel worksheet.

The experiment was designed in the way to investigate the equilibration time, effect of stirring $\left(\Delta E_{\text {stirr }}\right)$ and equilibrated values of SCrE potential $(E)$ after addition of titrant, Figure 4. Therefore, with each addition of base the magnetic stirrer was turned on for $200 \mathrm{~s}$ (blue points on Figure $4 ; E_{\text {stirron}}$ ) then turned off (red points on Figure $4 ; E_{\text {stirroff }}$ ), then again after 5 min turned on for $200 \mathrm{~s}$. The procedure was repeated. In the case of slow interfacial equilibration, a relatively stable reading is usually reached after a few minutes, but the obtained values do not necessarily correspond to equilibrium. This can only be assured by performing titrations in both directions to examine possible hysteresis. The problem can be solved by prolonged equilibration time or application of ultrasound. ${ }^{[9]}$

Form the results of the acid-base potentiometric titrations the effect of $\mathrm{pH}$ on inner surface potential for all metal oxide and fluorite $\mathrm{SCr}$ electrodes was examined. The values of the inner surface potential were calculated from the measured $\mathrm{SCr}$ electrode potentials using Eq. 7.

The effect of electrolyte concentration (at constant $\mathrm{pH}$ and stirring speed) on the measured electrode potential was investigated for all metal oxide and fluorite $\mathrm{SCr}$ electrodes using the same experimental set-up. The concentration of electrolyte was controlled by adding the corresponding inert electrolyte aqueous solution. ${ }^{[62-64]}$ After

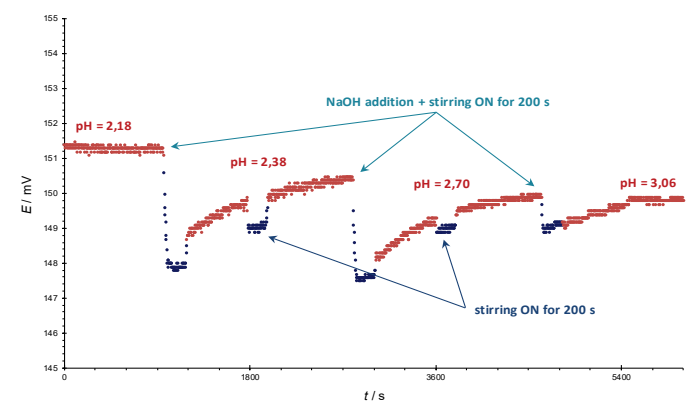

Figure 4. Enlargement of acidic part of Figure 3. Effect of stirring on measured electrode potential of SCrE hematite, $\alpha-\mathrm{Fe}_{2} \mathrm{O}_{3}(0001)$ electrode after addition of base with stirring off $(\bullet)$, and with stirring on $(\bullet)$. With each addition of base, the magnetic stirrer was turned on for $200 \mathrm{~s}$ (blue points) than turned off (red points), then again after 5 min turned on for $200 \mathrm{~s}$. The procedure was repeated. 


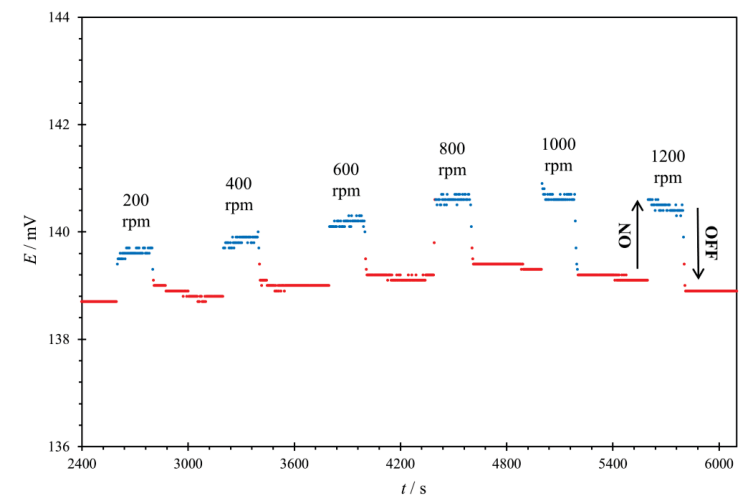

Figure 5. Influence of stirring speed on stirring potential of fluorite (111) SCrE. Time dependence of electromotivity of cell composed of fluorite SCrE and a reference electrode with spikes of different height as a result of changing stirring speed. Measurement was done under inert atmosphere (Ar) at $\mathrm{pH}=5.0 \pm 0.2,(25.0 \pm 0.1)^{\circ} \mathrm{C}$ and $I_{c} \approx 0.001 \mathrm{~mol} \mathrm{dm}^{-3}$ was adjusted by $\mathrm{NaCl}$ solution.

each addition of electrolyte solution magnetic stirrer (728 Magnetic Stirrer, Metrohm, Switzerland) was turned on at stirring speed about $600 \mathrm{rpm}$ for $200 \mathrm{~s}$ and then turned off. After the stabilization of the reading $(\mathrm{d} E / \mathrm{d} t<0.1 \mathrm{mV} / \mathrm{min})$, the procedure was repeated. It was found for all examined metal oxide and fluorite electrodes that increase of electrolyte concentration lower the absolute value of inner surface potential and decrease the effect of stirring on measured SCrE potential.

The effect of stirring speed (from 200 to $2000 \mathrm{rpm}$ ), at constant $\mathrm{pH}$ and ionic strength, on the measured electrode potential was investigated for all metal oxide and fluorite $\mathrm{SCr}$ electrodes using the same experimental set-up. The impact of the stirring speed on $\Delta E_{\text {stirr }}$ of fluorite (111) $\mathrm{SCrE}$, as the typical one is shown on Figure 5. Measurements with fluorite (111) SCrE were done at $\mathrm{pH}=5.0 \pm 0.2$ controled by addition of $0.1 \mathrm{~mol} \mathrm{dm}^{-3} \mathrm{HCl}$. During the experiments, stirring speed was initially adjusted to a certain value and then magnetic stirrer was turned on for $200 \mathrm{~s}$ (blue points on Figure 5; $\left.E_{\text {stirron}}\right)$, then turned off (red points on Figure $5 ; E_{\text {stirroff }}$ ). The procedure was repeated for different stirring speed. It was found for all examined metal oxide and fluorite electrodes that increase of stirring speed increase the effect of stirring on measured SCrE potential.

\section{The Construction of the High Impedance Voltmeter}

pure single crystals, especially artificial, tend to have minimum of current carriers (holes), so their electrical resistance can be high enough $\left(>10^{11} \Omega\right)$ to make measurements with commercial pH meters $\left(Z \approx 10^{12} \Omega\right)$ inaccurate and nearly impossible. One way of solving this

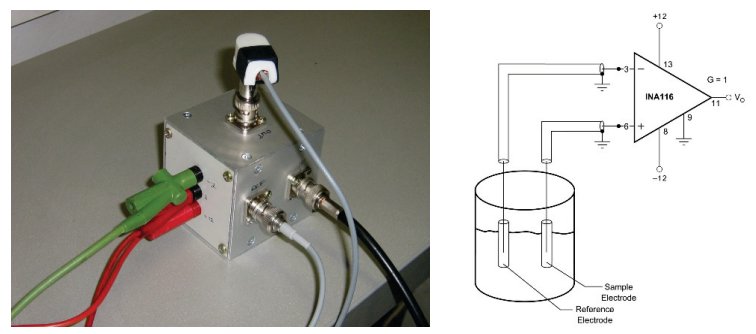

Figure 6. The high impedance voltmeter $\left(10^{15} \Omega\right)$ for measurements of the electrode potentials of high resistance single crystal electrode. The sensitivity is increased by using the amplifier INA116 (right).

problem is using voltmeters of impedances at least two orders of magnitude higher than the expected resistance of the measured circuit (which mainly comes from the crystal). The high impedance is achieved by using the amplifier INA116 (with declared impedance $10^{15} \Omega$ ) which makes it suitable for more precise measurements of potentials in circuits with high resistances $\left(R>10^{13} \Omega\right)$, Figure 6 . The sensibility of the potential measurements was improved by using high impedance voltmeter and Faraday's cage assembly for the whole measured system. The unavoidable noise level was generally assessed to be around $\pm 0.1 \mathrm{mV}$.

\section{Applying the External Voltage}

In order to investigate the mechanism of charging and equilibration at metal oxide/aqueous electrolyte interface the external voltage was applied in series with $\mathrm{SCrE}$ and resulting open circuit potential was measured. After addition of acid or base, at the metal oxide surface the distribution of potential determining ions takes place as well as formation of an electrified interface. This process has been described in literature in the following steps: ${ }^{[65]}$ distribution of ions, charging of electrolyte side of interface, charging of the metal oxide surface sites, development of interfacial potential and finally the equilibration. By adding an external voltage (battery) in series with SCrE the formation of an electrified interface conceives similar process. The system archives the equilibrium but additionally the polarization of the interface takes place. The resulting open circuit potential is measured by high impedance voltmeter. In the circuit, only the potential of the single crystal/aqueous electrolyte interface is sensitive to the concentration of potential determining ions. The equilibrium condition within the $\mathrm{SCrE} /$ aqueous electrolyte interface was tested by introducing an additional voltage source (battery; $U_{\mathrm{ex}}=1.5 \mathrm{~V}$ ) into the circuit. The introduced potentials were higher and lower than the electromotive force of the examined cell (SCrE-reference electrode) so that a current passed through the interface. The direction was also changed and the state at the interface was consequently shifted from its 
equilibrium state. The results of these tests showed that after equilibration the $\mathrm{SCrE}$ electrode potential remained the same. The small $\left(U_{\mathrm{ex}} \leq 0.20 \mathrm{~V}\right)$ disturbance did not have any effect, which confirmed that the system was sufficiently reversible and that a $\mathrm{pH}$-meter yields values that correspond to interfacial equilibrium. Higher external voltage causes the polarization of the electrodes as well as the irreversible transformation of metal oxide electrode surfaces. ${ }^{[33,34]}$

The electrical current in the electrical circuit with the cell assembled from a single crystal electrode and reference electrode during the equilibration was measured with a picoammeter. By integrating the function $i(t)$ and dividing by the surface area the surface charge density of the 0 - plane was evaluated. The obtained values of the surface charge density were in accordance with the data found in the literature. [33]

Though these measurements were somewhat inconclusive regarding direct determination of charge densities from charging/discharging curves (the processes turned out to be too fast for data collection), the measurements showed that this concept, with minor modifications, certainly has practical potential.

\section{Resistance of the Metal Oxide Single Crystals}

Electrical resistance of the SCrE and electrical current was measured using a Keithley 2401 SourceMeter. The resistances of the SCrEs were measured "on dry", by connecting the crystal directly to the instrument. The good electric contact with the crystal was obtained by applying the silver particles based conductive paint on the two opposite surfaces of the crystal, and connecting them to the instrument's leads. The measurement of resistivity as a crystal's property was not aim here because there is very little data in literature for comparison, as resistivity greatly depends on impurities and synthesis methods. The main reason for resistance measurements was assessment of specific crystals' applicability for the surface potential measurements.

The measured values of the crystals resistance also suggested that some crystals have anisotropic electrical

Table 2. The measured resistances of the SCrEs.

\begin{tabular}{cc}
\hline SINGLE CRYSTAL & $R / \mathrm{G} \Omega$ (exp.) \\
\hline rutile, $\mathrm{TiO}_{2}(100)$ & 24.6 \\
rutile, $\mathrm{TiO}_{2}(010)$ & 50.1 \\
hematite, $\mathrm{Fe}_{2} \mathrm{O}_{3}(001)$ & $>1000$ \\
ceria (111) & $>1000$ \\
ceria (100) & $>1000$ \\
$\mathrm{AgCl}$ & $4.5 \times 10^{-4}$ \\
sapphire, $\mathrm{Al}_{2} \mathrm{O}_{3}(0001)$ & $>1000$ \\
\hline
\end{tabular}

properties (rutile, $\mathrm{TiO}_{2}$ ), which is in complies to previous researches. ${ }^{[66]}$ This fact might be needed to be considered when comparing measurements between the same sort of electrodes of different crystallographic orientation.

Additionally, it was found that the shape of the increase/decrease of measured SCrE potential during titration varies depending of resistance, showing more or less noticeable temporary polarization of electrode during equilibration. This might be related to the mechanism of current propagation through crystal.

\section{RESULTS AND DISCUSSION}

The electrical properties, limits and the sensibility of metal oxide single crystal electrodes (hematite, ceria, sapphire and rutile) were examined. All SCrE exhibit stable response and satisfying reproducibility. Measured electrical resistance of the metal oxide $\mathrm{SCrE}$ (between $10^{10} \Omega$ and $10^{13}$ $\Omega$ ) depends on the type and orientation of the crystal. Electric current may be propagated through crystals in three ways: due to electronic, electrolytic and dielectric conduction. ${ }^{[67]}$ The first is the normal type of current flow in conductors such as metals containing free electrons. In an electrolyte solution the current is carried by ions. Usually, apart from solutions, such conductivity is found within doped semiconductors, and is a result of presence of foreign ions within crystal lattice. Dielectric conduction occurs within insulators, which have very small number of free carriers or none at all. Within the external electric field, the electrons are slightly displaced from their equilibrium positions, i.e. the separation of negative and positive charges occurs, which is known as dielectric polarization. It produces a so-called displacement current. Measurement of the SCrE surface potentials is possible when current flows through the crystal body. In most similar investigations, i.e. Ref. [68] the doped crystals with higher conductivity were used, therefore measurements were easier and reliable. In our measurements, the used crystals were as pure as possible, which means that otherwise neglected electrical properties of crystals (conductivity, dielectricity) can influence the measurements and interpretation of the results. However, our methodology for electrode potentials measurements was based on $\mathrm{pH}$-meters used as high impedance voltmeters $\left(\sim 10^{12} \Omega\right)$ and constructed high impedance voltmeter with amplifier INA11 $\left(\sim 10^{15} \Omega\right)$ which makes them suitable for measuring electrode potential of the crystals with resistances up to $10^{10} \Omega$ or up to $10^{13} \Omega$, respectively. Higher resistances would yield in unacceptable erroneous values. However, experiments showed that even with crystals that are practically insulators, the valid and repeatable results are still obtained. It can be concluded that in some cases, dielectric conduction within the metal oxides occurred. It is noticed that due to extremely low currents in 
circuit, the difference in conductivity mechanism does not influence measurements of the equilibrium surface potentials. However, some of the important factors in calculating thermodynamic parameters and electrokinetic potential from measured surface potentials are electrical capacitances of the interfacial layers (usually estimated or assessed by different experimental techniques, such as electrochemical impedance spectroscopy). Apart from double layer capacitance, the crystal's capacitance might play a big role and should not be omitted.

During potentiometric measurement $\mathrm{SCr}$, glass and reference electrodes are immersed in the aqueous electroIyte solution. Addition of titrants yields to the certain $\mathrm{pH}$ and equilibration at the surface of the $\mathrm{SCr}$ electrode. Switching on and off the magnetic stirrer results in sudden change of measured electrode potential. The effect is reversible. After certain equilibration time electrode potential reaches the new constant equilibrium value. Similar influence of stirring was found in previous investigations for glass electrode. ${ }^{[59-61]}$ The effect of liquid flow along a solid quartz and fluorite surfaces, was also observed during surface-specific sum frequency generation spectroscopy. ${ }^{[69]}$ For the purposes of the better understanding of the mechanisms contributing to the development of the electrode potentials and the description of the charging mechanisms at the electrical interfacial layer two kinds of experiments were performed. The effect of stirring $\left(\Delta E_{\text {stirr, }}\right.$ equation 8) was investigated as a function of $\mathrm{pH}$ (at constant ionic strength), as a function of ionic strength (at constant $\mathrm{pH}$ ) and as a function of stirring speed (at constant $\mathrm{pH}$ and ionic strength). At the Figure 7. the dependency of

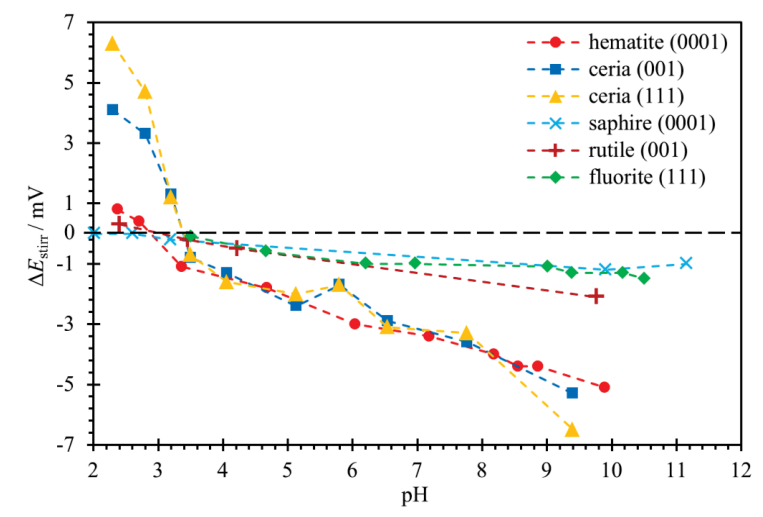

Figure 7. The effect of stirring on hematite $(0001)(\bullet)$, ceria (001) (匹), ceria (111) ( 4 ), saphire (0001) (x), rutile (001) (+) and fluorite (111) ( ) single crystal electrodes as a function of $\mathrm{pH}$. The $\mathrm{pH}$ was controlled by adding $0.1 \mathrm{~mol} \mathrm{dm}^{-3} \mathrm{NaOH}$ to the $0.01 \mathrm{~mol} \mathrm{dm}^{-3} \mathrm{HCl}$ solution. During measurements, the aqueous electrolyte solution was mixed with a magnetic stirrer, thermostated at $(25.0 \pm 0.1)^{\circ} \mathrm{C}$ and kept under argon atmosphere. the stirring potential on the hematite (0001), ceria (001), ceria (111), saphire (0001), rutile (001) and fluorite (111) single crystal electrodes on $\mathrm{pH}$ is presented. $\mathrm{pH}$ is measured with the glass electrode which is also affected by stirring, but experiments showed that this change is not significant enough to impact measured $\mathrm{pH}$ values.

The following results were observed: (i) immediately after addition sudden decrease (in the acidic region) or sudden increase (in the basic region) of measured $E$ values occur; (ii) switching on and off the magnetic stirrer influence the measured $E$ value, the difference depends on $\mathrm{pH}$, ionic strength and stirring speed; (iii) the direction of the titration (addition of base or acid) do not affect the value of $\Delta E_{\text {stirr }}$ but influence the time of equilibration (slow equilibration if $\mathrm{pH}<\mathrm{pH}_{\text {iep }}$ and fast equilibration if $\mathrm{pH}>\mathrm{pH}_{\text {iep }}$ ); (iv) the fast equilibration after switching off the magnetic stirrer in the basic region compared to the time of the equilibration in the acidic region indicates that the rate of the surface protonation is not the same in the acidic and basic region. The long equilibration time shows that equilibration within EIL is not only diffusion controlled process.

These observations coupled with measurements of electrical resistance and electrical current lead to conclusions about charge transfer, ion distribution and water rearrangement near the electrode surface. Immediately after addition of acid or base and during equilibration, even using a high impedance instrument, a current passes through the interface and temporary polarization may occur. Switching on the magnetic stirrer reversibly alters the interfacial equilibrium.

In the case of soluble materials like fluorite $\left(K_{s p}^{\circ}=\right.$ $\left.3.08 \times 10^{-11}\right)^{[70]}$ the dissolution of the crystal plane should also be consider. The rate of dissolution is expected to be faster due to vigorous stirring and liquid flow among the crystal plane. Interestingly, the effect of stirring observed for the fluorite (111) SCrE was similar as the results obtained for the examined metal oxides whose dissolution under the measured condition are negligible, Figure 7. Furthermore, it was found that the direction of the titration does not affect the value of stirring potential. As was predicted by Lis et $a^{\left[{ }^{[71]}\right.}$ in the presence of stirring the surface of (111) fluorite becomes more positively charged for almost all examined $\mathrm{pH}$ values.

The point of zero stirring potential $\left(\mathrm{pH}_{\text {stirr }}\right)$ at which $\Delta E_{\text {stirr }}=0$ for all investigated metal oxide single crystal and fluorite electrode was found to be in the narrow $\mathrm{pH}$ region between $\mathrm{pH}=2$ and $\mathrm{pH}=3.3$ (Figure 7). At $\mathrm{pH}>\mathrm{pH}_{\text {stirr }}$ the increase of measured $E$ signal was obtained during stirring. While in the acidic region, the opposite effect was noticed. The value of $\mathrm{pH}_{\text {stirr }}$ should not be confused with $\mathrm{pH}_{\text {pzp }}$ of crystal itself which is defined by the thermodynamic equilibrium constants of interfacial reactions (Equation 1). For most investigated metal oxide crystal planes ${ }^{[37,38,40,41]}$ the 


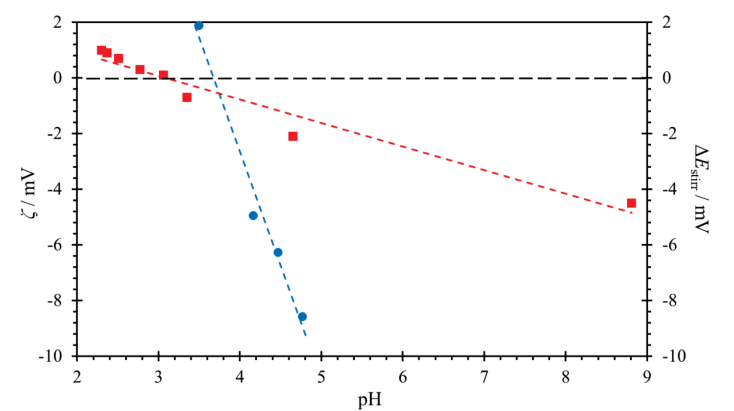

Figure 8. Comparison between electrokinetical (streaming) potential (blue $\bullet$ ), change in potentials upon stirring ( $\mathrm{red} \mathbf{m}$ ) during measurements with hematite (0001) single crystal electrode. Ionic strength was $I_{c} \approx 0.01 \mathrm{~mol} \mathrm{dm}^{-3}$ and controlled with $\mathrm{NaCl}$, thermostated at $(25.0 \pm 0.1){ }^{\circ} \mathrm{C}$ and kept under argon atmosphere.

broad electroneutrality region were obtained in which case the accurate value of $\mathrm{pH}_{\mathrm{pzp}}$ was hard to obtained, but rather $\mathrm{pH}_{\mathrm{pzp}}$ zone.

The value of $\mathrm{pH}_{\text {iep }}$ for the same metal oxides crystal planes obtained from streaming potential results is found to be in the narrow $\mathrm{pH}$ region between $\mathrm{pH}=2.0$ and $\mathrm{pH}=$ 4.5 (Table 3). Similar to metal oxide $\mathrm{SCr}$ electrodes the $\mathrm{pH}_{\text {stirr }}$ for fluorite (111) $\mathrm{SCrE}$ was found to be in the $\mathrm{pH}$ region between $\mathrm{pH}=3$ and $\mathrm{pH}=4$ (Figure 7) which coincides with isoelectric point of fluorite colloids particles determined at the similar experimental conditions $\left(\mathrm{pH}_{\text {iep }}=\right.$ 3.6). ${ }^{[63]}$ The origins of both, stirring potential as well as electrokinetic streaming potential, are in the distribution of ions in the diffuse part of the EIL.

On the relative scale, for all investigated metal oxide surfaces the stirring potential follows the expected trend of electrokinetic $\zeta$-potential obtained by means of streaming potential measurements. ${ }^{[72]}$ At Figure 8 the stirring potential data obtained for hematite (0001) SCrE was compared with streaming potential measurement. The points of zero stirring potential of examined crystal planes were compared with isoelectric points of the same crystal planes and with points of zero potential obtained for the colloid particles of same materials (Table 3).
It is obvious that the isoelectric points coincide with the $\mathrm{pH}$ region where the smallest effect of stirring was observed $\mathrm{pH}_{\text {stirr }}\left(\Delta E_{\text {stirr }}=0\right) \approx \mathrm{pH}_{\text {iep }}$. As in the case of electrokinetic potential increasing of electrolyte concentration decreases the value of measured $\Delta E_{\text {stirr }}$ (Figure 9).

There could be several reasons for this kind of result. On the one hand solubility of $\mathrm{CaF}_{2}$ increases as ionic strength increases, but on the other hand the effect of the ionic strength on the propagation of charge at the fluorite (111) surface is unknown. Another way to look at this problem is to imagine that with increasing ionic strength the electrical interface layer becomes more ordered so more energy (for example the increase of the stirring speed) is needed to introduce new water molecules near the surface and to disperse near-surface dissolved $\mathrm{Ca}^{2+}$ and $\mathrm{F}^{-}$ions into the bulk of fluid. As a result of that is biasing the dissolution equilibria toward the release of ions from fluorite surface as was suggested by Lis et al[71]

The obtained results suggest that the similar processes take place during the liquid flow among a solid surface of SCrE on electrode potential, as during the streaming potential measurements ${ }^{[72]}$ and the surface-specific sum frequency generation spectroscopy. ${ }^{[69]}$ The length over which the electrical properties of the metal oxide and fluorite surfaces extends in the electrolyte solutions depends on the surface charge density, the ionic strength and rearrangements of the water molecules.

The influence of stirring speed on the $\mathrm{SCr}$ electrode potential was also examined (Figure 5 and Figure 10). It was found that the $\Delta E_{\text {stirr }}$ is proportional to the stirring speed. However, a certain minimum speed at which no effect is observed (<100 rpm), as well as maximum speed $(>1000$ $\mathrm{rpm}$ ) after which no change in $\Delta E_{\text {stirr }}$ occurs.

These findings indicate that the effect of stirring is the consequence of the redistribution of ions within electrical interfacial layer. The potential developed at the certain distance from the metal oxide and fluorite surface is under the influence of accumulated and weakly bounded ions in diffuse part of electrical interfacial layer which surface concentrations depend of the stirring speed. During $\mathrm{SCrE}$ potential measurements it was noticed that some

Table 3. The point of zero stirring potential and point of zero potential of the SCrEs in respect to the reference $\mathrm{Ag} / \mathrm{AgCl}$ $\left(3 \mathrm{~mol} \mathrm{dm}^{-3} \mathrm{KCl}\right)$ electrode were measured in aqueous $\mathrm{NaCl}$ solution $\left(c=0.01 \mathrm{~mol} \mathrm{dm}^{-3}\right)$.

\begin{tabular}{cccc}
\hline SINGLE CRYSTAL & $\mathrm{pH}_{\text {stirr }}\left(\Delta E_{\text {stirr }}=0\right)$ & $\mathrm{pH}_{\text {pzp }}\left(\psi_{0}=0\right)$ & $\mathrm{pH}$ iep $(\zeta=0)$ \\
\hline rutile, $\mathrm{TiO}_{2}(001)$ & 2.5 & $6^{[72]}$ & $5.6^{[72]}$ \\
hematite, $\mathrm{Fe}_{2} \mathrm{O}_{3}(0001)$ & 3.0 & $8^{[141]}$ & $3.5-4.0^{[72]}$ \\
sapphire, $\mathrm{Al}_{2} \mathrm{O}_{3}(0001)$ & 2.3 & $6.5^{[72]}$ & $4.1^{[73]}$ \\
ceria, $\mathrm{CeO}_{2}(111)$ & 3.4 & $5.9^{[74]}$ & \\
\hline
\end{tabular}




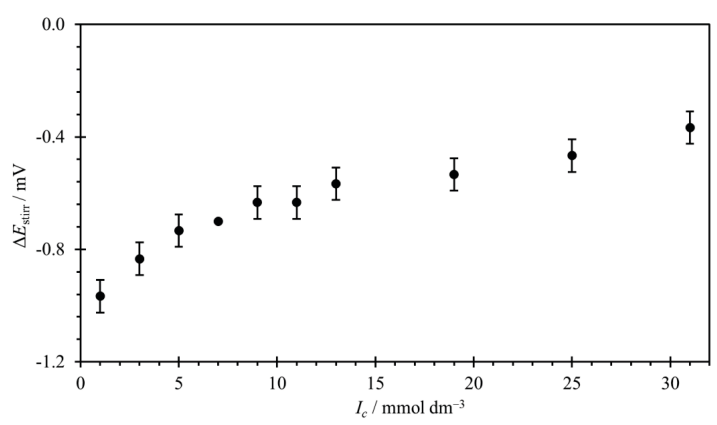

Figure 9. The effect of stirring on fluorite (111) SCrE as a function of ionic strength controlled by $\mathrm{NaCl}$ solution. Measurements were done under inert atmosphere $(\mathrm{Ar}) \mathrm{at} \mathrm{pH}$ $=5.0 \pm 0.2,(25.0 \pm 0.1)^{\circ} \mathrm{C}$ and stirring speed about $600 \mathrm{rpm}$.

electrodes behave generally differently than another, especially in terms of time of equilibrium, and hysteresis which sometimes occurs upon two-way titration. Such behavior might be a consequence of the electrical and structural properties of the crystals which define the distribution of the ions within the EIL, as well as the kinetics of surface reactions.

\section{CONCLUSION}

The values of the inner surface potentials of several metal oxide crystals (hematite, ceria, sapphire and rutile) and fluorite crystal were obtained, by means of single crystal electrodes, as a function of $\mathrm{pH}$ and ionic strength. The special attention was paid to the changes of electrode potentials observed when magnetic stirring was turned on and off. The contact of the electrolyte solution with the crystal surfaces causes the formation of the amphoteric surface groups and reactions of these surface groups with the potential determining ions. The surface becomes electrically charged, whiles within the interfacial region the equilibrium distribution of counterions and reorientation of water dipoles occur. The equilibrium state at the metal oxide interface, determined by the thermodynamic equilibrium constant, can be disturbed by addition of acid, base or salt in the electrolyte solution. Addition of titrant leads to the surface reactions of the charged surface groups, and to the new distribution of counterions and reorientation of water molecules. A new equilibrium establishes. During the equilibration process the surface of a single crystal electrode temporary polarizes which is recorded as a steep shift of the measured electrode signal immediately after addition of titrant. Additionally, the nanoampere electrical current was measured by a highly sensitive multimeter. The experiments with additional voltage $(<200 \mathrm{mV})$ in the open circuit indicate that the reversible equilibration takes place.

The effect of liquid flow along the solid surface was tested by switching the magnetic stirrer on and off. If the

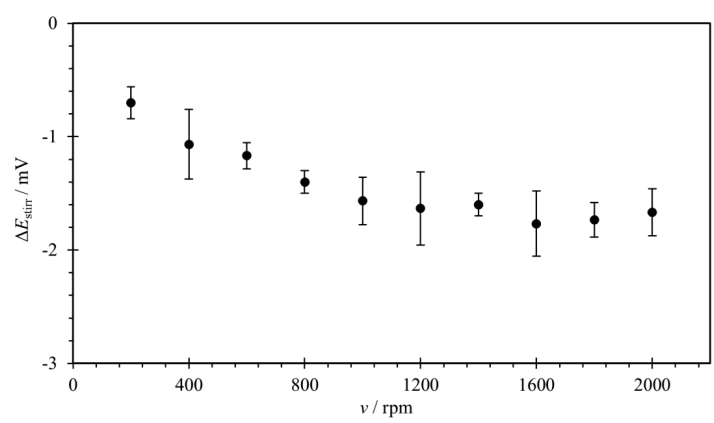

Figure 10. The effect of stirring on fluorite (111) single crystal electrode as a function of the stirring speed adjusted by a magnetic stirrer. Measurements were done under inert atmosphere (Ar) at pH $=5.0 \pm 0.2,(25.0 \pm 0.1){ }^{\circ} \mathrm{C}$ and $I_{c} \approx$ $0.001 \mathrm{~mol} \mathrm{dm}^{-3}$.

metal oxide surfaces are positively charged by switching on the stirrer the surface potential becomes more negative indicating that weakly bounded counterions in the diffusion part of EIL and oriented water molecules are affected. The local change of concentration of the potential determining ions affects the surface equilibrium reaction (1). In the acidic region the dominant ions in the bulk of the solution as well as in the interfacial region are $\mathrm{H}^{+}$ions. Due to vigorous stirring the local concentration of $\mathrm{H}^{+}$ions decreases, forming more $\equiv \mathrm{MO}^{z}$ surface groups and establishing the new equilibrium state. By switching off the magnetic stirrer the system quickly returns to the original equilibrium state. On the contrary in the basic region, the dominant ions in the bulk of the solution as well as in the interfacial region are $\mathrm{OH}^{-}$ions. Vigorous stirring decreases the local concentration of $\mathrm{OH}^{-}$ions, more $\equiv \mathrm{MOH}^{2+1}$ are formed and surface potential becomes more positive. The shape of the stirring potential curves and the point of zero stirring potential coincide with isoelectric point and electrokinetic potentials obtained by streaming potential techniques. The slow equilibration is a consequence of fast diffusion of ions and slow surface reactions. In the case of soluble and more reactive surfaces, like fluorite, the dissolution and surface transformations should not be neglected. These findings are expected to have an impact on the understanding and modeling of interfacial complexation and may possibly explain the difference in results obtained for colloid particles and flat surfaces of the same material.

Acknowledgment. This work has been supported by Croatian Science Foundation under the project (IP-2014-09-6972). The authors wish to acknowledge Saša Blažeka who helped in construction of the high impedance voltmeter for measurement the electrode potential of high resistance single crystal electrode. One of the authors (D. Namjesnik) stated that he was not involved in editorial decisions related to this manuscript. 


\section{REFERENCES}

[1] A. V. Delgado, F. González-Caballero, R. J. Hunter, L. K. Koopal, J. Lyklema, Measurement and Interpretation of Electrokinetic Phenomena (IUPAC Technical Report). Pure Appl. Chem. 2005, 77, 1753.

[2] J. Lyklema, Fundamentals of interface and colloid science. Volume 2, Solid-liquid interfaces. $1^{\text {st }} \mathrm{ed}$. 1995 (Academic Press: London)

[3] Y. -W. Chen, I. -H. Chu, Y. Wang, H. -P. Cheng, Phys. Rev. B 2011, 84, 155444.

[4] D. E. Yates, S. Levine, T. W. Healy, J. Chem. Soc. Faraday Trans. 1 Phys. Chem. Condens. Phases 1974, 70, 1807.

[5] W. Stumm, C. P. Huang, S. R. Jenkins, Croat. Chem. Acta 1970, 42, 223.

[6] W. Stumm, L. Sigg, B. Sulzberger, Chemistry of the solid-water interface: processes at the mineralwater and particle-water interface in natural systems. Wiley, 1992.

[7] N. Kallay, S. Žalac, and D. Kovačević, Thermodynamics of the Solid/Liquid Interface. Its Application to Adsorption and Colloid Stability in Surface Complexation Modelling (Ed.: J. Lützenkirchen) in the series Interface Science and Technology, Elsevier, 2006.

[8] N. Kallay, T. Preočanin, D. Kovačević, J. Lutzenkirchen, E. Chibowski, Croat. Chem. Acta 2010, 83, 357.

[9] T. Preočanin, A. Čop, N. Kallay, J. Colloid Interface Sci. 2006, 299, 772.

[10] P. Zarzycki, S. Chatman, T. Preočanin, K. M. Rosso, Langmuir 2011, 27, 7986.

[11] P. Zarzycki, T. Preočanin, J. Colloid Interface Sci. 2012, 370, 139.

[12] E. Kinoshita, F. Ingman, G. Edwall, S. Glab, Electrochim. Acta 1986, 31, 29.

[13] S. Ardizzone, A. Carugati, S. Trasatti, Properties of thermally prepared iridium dioxide electrodes. Elsevier, 1981.

[14] S. Ardizzone, M. Radaelli, Open-circuit potentials of $\mathrm{ZrO}_{2}$ electrodes. Elsevier, 1989.

[15] M. J. Avena, O. R. Cámara, C. P. De Pauli, Colloids and Surfaces 1993, 69, 217.

[16] N. H. G. Penners, L. K. Koopal, J. Lyklema, Colloids and Surfaces 1986, 21, 457.

[17] R. G. Bates, J. B. Macaskill, R. G. Bates, Pure Appl. Chem. 1978, 50, 1701.

[18] J. F. Schneck, J. Colloid Interface Sci. 1977, 61, 569.

[19] C. Chicos, T. H. Geidel, Colloid Polym. Sci. 1983, 261, 947.

[20] L. Bousse, N. F. De Rooij, P. Bergveld, Surf. Sci. 1983, 135, 479.

[21] W. M. Siu, R. S. C. Cobbold, IEE Trans. Electron Devices 1979, ED-26, 1805.
[22] R. E. G. Hal, J. C. T. Eijkel, P. Bergveld, Adv. Colloid. Interface Sci. 1996, 69, 31.

[23] P. Bergveld, IEEE Trans. Biomed. Eng. 1970, BME-17, 70.

[24] J.-C. Chou, L. P. Liao, Thin Solid Films 2005, 476, 157.

[25] B. Lübbers, A. Schober, Chem. Anal. (Warsaw) 2009, 54, 1121.

[26] N. Kallay, Z. Dojnović, A. Čop, J. Colloid Interface Sci. 2005, 286, 610.

[27] N. Kallay, T. Preočanin, T. Ivšić, J. Colloid Interface Sci. 2007, 309, 21.

[28] T. Preočanin, W. Janusz, N. Kallay, Colloids Surfaces A Physicochem. Eng. Asp. 2007, 297, 30.

[29] T. Preočanin, M. Tuksar, N. Kallay, Appl. Surf. Sci. 2007, 253, 5797.

[30] N. Kallay, T. Preočanin, J. Marković, D. Kovačević, Colloids Surf., A 2007, 306, 40.

[31] D. Kovačević, D. Mazur, T. Preočanin, N. Kallay, Adsorption 2010, 16, 405.

[32] N. Kallay, T. Preočanin, J. Colloid Interface Sci. 2008, 318, 290.

[33] K. Shimizu, J. -F. Boily, J. Phys. Chem. C 2015, 119, 5988.

[34] K. Shimizu, J. Nystrom, P. Geladi, B. Lindholm-Sethson, J. -F. Boily, Phys. Chem. Chem. Phys. 2015, 17, 11560.

[35] J. -F. Boily, S. Chatman, K. M. Rosso, Geochim. Cosmochim. Acta 2011, 75, 4113.

[36] K. Shimizu, A. Lasia, J. -F. Boily, Langmuir 2012, 28, 7914.

[37] S. V Yanina, K. M. Rosso, Science 2008, 320, 218.

[38] S. Chatman, P. Zarzycki, K. M. Rosso, Phys. Chem. Chem. Phys. 2013, 15, 13911.

[39] S. Chatman, P. Zarzycki, K. M. Rosso, ACS Appl. Mater. Interfaces 2015, 7, 1550.

[40] N. Kallay, T. Preočanin, M. Sapunar, D. Namjesnik, Surf. Innov. 2014, 2, 142.

[41] J. Lutzenkirchen, F. Heberling, F. Šupljika, T. Preočanin, N. Kallay, F. Johann, Faraday Discuss. 2015, 180, 55.

[42] M. Lucas, J. -F. Boily, Langmuir 2015, 31, 13618.

[43] N. Kallay, D. Čakara, J. Colloid Interface Sci. 2000, $232,81$.

[44] N. Kallay, A. Čop, E. Chibowski, L. Holysz, J. Colloid Interface Sci. 2003, 259, 89.

[45] D. Harvey, Modern Analytical Chemistry. McGraw Hill, 2000.

[46] R. K. Quinn, R. D. Nasby, R. J. Baughman, Mater. Res. Bull. 1976, 11, 1011.

[47] N. Kallay, F. Šupljika, T. Preočanin, J. Colloid Interface Sci. 2008, 327, 384.

[48] T. Preočanin, F. Šupljika, N. Kallay, J. Colloid Interface Sci. 2009, 337, 501.

[49] T. Hiemstra, Langmuir 2012, 28, 15614. 
[50] T. Preočanin, F. Šupljika, N. Kallay, J. Colloid Interface Sci. 2011, 354, 318.

[51] T. Preočanin, F. Šupljika, N. Kallay, J. Colloid Interface Sci. 2010, 346, 222.

[52] I. Mills, T. Cvitaš, K. Homann, N. Kallay, K. Kuchitsu, Quantities, Units and Symbols in Physical Chemistry; $2^{\text {nd }}$ Edition. Blackwell, Oxford, 1993.

[53] T. Preočanin, N. Kallay, Adsorption 2013, 19, 259.

[54] G. A. Parks, P. L. de Bruyn, J. Phys. Chem. 1962, 66, 967.

[55] J. S. Noh, J. A. Schwarz, J. Colloid Interface Sci. 1989, 130, 157.

[56] S. Žalac, N. Kallay, J. Colloid Interface Sci. 1992, 149, 233.

[57] C. Werner, H. Körber, R. Zimmermann, S. Dukhin, H. J. Jacobasch, J Colloid Interface Sci. 1998, 208, 329.

[58] P. J. Scales, F. Grieser, T. W. Healy, L. R. White, D. Y. C. Chan, Langmuir 1992, 8, 965.

[59] K. L. Cheng, J. Colloid Interface Sci. 2001, 239, 385.

[60] K. L. Cheng, Da-Ming Zhu, Sensors 2005, 5, 209.

[61] Z. Galbács, H. Van Langenhove, G. Galbácsa, Talanta 2005, 66, 809.

[62] J. D. Miller, J. B. Hiskey, J. Colloid Interface Sci. 1972, 41, 567.
[63] A. Uçar, H. Özdağ, Trans. Instn. Min. Metall. (Sec. C: Mineral Process. Extr. Metall.), 2002, 111, 100.

[64] L. Wu, W. Forsling, J. Colloid Interface Sci. 1995, 174, 178.

[65] J. O'M. Bockris, A. K. N. Reddy, M. E. GamboaAldeco, Modern Electrochemistry Vol IlaFundamentals of Electrodics, Springer US, 2000.

[66] O. Byl, J. T. Yates, Jr., J. Phys. Chem. B 2006, 110 (46), 22966.

[67] William Lowrie, Fundamentals of Geophysics, $2^{\text {nd }}$ Edition, Cambridge University Press, 2007, p. 254.

[68] N. Hara, K. Sugimoto. J. Electrochem. Soc. 1990, 137(8), 2517.

[69] D. Lis, E. H. G. Backus, J. Hunger, S. H. Parekh, M. Bonn, Science 2014, 344, 6188.

[70] A. Garand, A. Mucci, Mar. Chem. 2004, 91, 27.

[71] D. Lis, E. H. G. Backus, J. Hunger, S. H. Parekh, M. Bonn, Science 344 (2014) 1138.

[72] T. Preočanin, D. Namjesnik, M. A. Brown, J. Lützenkirchen, Enviromental Chemistry 2017, 14(5), 295.

[73] S. V. Shevkunov, Dokl. Phys. 2005, 50, 234.

[74] D. Namjesnik, S. Mutka, D. Iveković, A. Gajović, M. Willinger, T. Preočanin, Adsorption 2016, 22, 825. 